\title{
Pedagogización de la sociedad y crisis de la educación. Elementos para una critica de la(s) critica(s)
}

\section{Pedagogicalization of Society and the Crisis of Education. Elements for a Criticism of Criticism(s)}

Pedagogização da sociedade e crise da educação. Elementos para uma crítica da(s) crítica(s)

\section{Carlos Ernesto Noguera-Ramírez* Gustavo Adolfo Parra**}

* Doctor en Educación Universidad Federal do Rio Grande do Sul-ufrgs. Profesor titular y Decano de la Facultad de Educación de la Universidad Pedagógica Nacional de Colombia. Miembro del Grupo de Historia de la Práctica Pedagógica (GHPP). e-mail: cnoguera@pedagogica.edu.co

** Licenciado en Matemáticas Universidad Pedagógica Nacional, estudiante de la Maestría en Educación Universidad Pedagógica Nacional de Colombia. Profesor Facultad de Educación, Universidad Pedagógica Nacional de Colombia. Miembro del Grupo de Historia de la Práctica Pedagógica (GHPP). e-mail: gaparral@pedagogica.edu.co 


\section{Introducción}

$\mathrm{E}$ n el momento actual, en el que la formación de un pensamiento crítico ha dejado de ser consigna revolucionaria y se ha convertido en un propósito común de cualquier programa académico, compartido por instituciones gubernamentales, organismos de cooperación, movimientos políticos diversos, organizaciones no gubernamentales, entre otros, proponer una crítica no resulta algo sorprendente. Por el contrario, particularmente en el marco de una institución universitaria de carácter oficial como la nuestra, resulta una propuesta casi obvia, esperable y quizás esperada.

Pareciera que la crítica está en el orden del día. Ser crítico, criticar, tomar posturas críticas, tener un pensamiento crítico no solo es algo deseable sino casi una exigencia, por lo menos en el espacio académico. Podría decirse, entonces, que con este texto se ratifica nuestra condición de académicos, de profesores de una universidad calificada como "pública"; en fin, estamos en el lugar adecuado y haciendo lo que se espera que debe hacerse. Sin embargo, hay algo de incómodo en el subtítulo, o mejor aún, en el problema mismo que nos hemos planteado para desarrollar en este texto: ¿acaso no hay ya suficientes críticas a la pedagogía? El texto entonces, ¿se propone retomar o presentar esas críticas? ¿Es un artículo sobre o desde las "pedagogías críticas"? ¿Es posible una nueva crítica, una crítica diferente a la ya tan criticada pedagogía?

Como verán, la situación en la que nos hemos puesto no es propiamente cómoda. Pero debemos decir, en verdad, que no escogimos voluntariamente ese lugar; más bien, fuimos llevados hasta allí por efecto de ciertas preguntas y análisis en los que hemos intervenido durante los últimos años. En un momento inicial se pensó en abandonar la idea de emprender una crítica, hasta se consideró la posibilidad de rechazar cualquier actitud crítica en vista de la existencia de tanta crítica, de tanto pensamiento crítico, de tantas teorías críticas. Pero el ejercicio del entendimiento nos llevó a percibir que esa era una vana posibilidad, o mejor, una imposibilidad. No es posible abandonar la crítica sin renunciar a la condición misma del pensamiento. La opción que quedaba era, entonces, intentar una crítica más sobre un asunto muy - o por lo menos bastante-criticado.

\section{¿Qué es la crítica?}

En primer lugar hay que decir que este texto no pretende ser ni una especie de pedagogía crítica ni una crítica de la(s) pedagogía(s). No se trata de una crítica cuyo propósito sea develar los errores, las incoherencias, las debilidades conceptuales, teóricas o epistemológicas de la pedagogía o de ciertas pedagogías; tampoco se trata de una crítica entendida como denuncia de las relaciones de dominación que impone toda pedagogía o cierta pedagogía (la tradicional o la burguesa). Algunos colegas brasileños (Silva, 1993; Veiga-Neto, 1997) hablaron de una postura "pós-crítica", es decir, de una perspectiva que, sustentada en las elaboraciones posestructuralistas y, particularmente, en la llamada "filosofía de la diferencia", pretende ir más allá de las críticas tanto neoliberales como marxistas a la educación y la pedagogía moderna. Tampoco es esa nuestra opción. ¿De qué se trata entonces? La crítica que se ha puesto en acción no pretende ser la verdadera crítica aunque, desde luego, sea considerada mejor que otras opciones en el sentido de sus efectos y posibilidades. Se trata de una perspectiva que parte del trabajo de Michel Foucault y que podría localizarse, contrario a quienes piensan que se trata de una mirada posmoderna, en la propia tradición de la modernidad, aún más, en la tradición abierta por Kant a propósito de su pregunta sobre “¿Qué es la Ilustración?”.

Foucault inscribe la crítica como un asunto propiamente moderno y, particularmente, señala que se trata de una actitud que aparece como contrapartida, como contracara de lo que llamó el proceso de "gubernamentalización", iniciado en Europa hacia los siglos XV y XVI. En otras palabras, ante la expansión y el desarrollo de un arte de gobernar a los seres humanos, aparece como su correlato una actitud moral y política, una manera de pensar que se plantea la cuestión de cómo no ser gobernado. Y aquí aclara rápidamente Foucault que con ello no quiere decir que a la gubernamentalización se habría opuesto, en un cara a cara, la afirmación contraria, es decir, un "no queremos ser gobernados en absoluto". Lo que quiere decir es que ante la inquietud acerca de la manera de gobernar y en la búsqueda de maneras de hacerlo, es posible localizar una cuestión que sería: "cómo no ser gobernados de esa forma, por ése, en nombre de esos principios, en vista de tales objetivos y por medio de tales procedimientos, no de esa forma, no para eso, no por ellos" (Foucault, 2007, pp. 7-8).

No es una recusa de cualquier o de toda forma de gobierno, sino una actitud que cuestiona el ser gobernado de determinadas formas, con ciertos fines y por ciertos sujetos. La Reforma del siglo XVI recoge una de las primeras manifestaciones de esa actitud crítica que se inició siglos antes y en cuyo centro estaba un cuestionamiento a la forma de gobierno de la Iglesia católica que llevó a la construcción de nuevas formas de conducción y dirección, de ahí 
que Foucault afirme que la crítica es históricamente bíblica, pues fue en la interpretación de las Escrituras en donde se definió esa actitud que llevó a millones de personas a rechazar una forma de gobierno.

En últimas, la crítica sería:

... el movimiento por el cual el sujeto se atribuye el derecho de interrogar a la verdad acerca de sus efectos de poder y al poder acerca de sus discursos de verdad; la crítica será el arte de la inservidumbre voluntaria, de la indocilidad reflexiva. La crítica tendría esencialmente como función la desujeción en el juego de lo que se podría denominar, con una palabra, la política de la verdad. (Foucault, 1995, pp. 7-8)

Se verá que esta definición que propone Foucault no está lejos de lo que Kant planteaba en 1784 con su pregunta “QQué es la ilustración?”. Recordemos que en ese texto periodístico Kant reconocía que la humanidad se encontraba autoritariamente en un cierto estado de minoría de edad caracterizado por su incapacidad de servirse de su propio entendimiento sin la dirección de otro, todo ello como resultado de una falta de decisión o de coraje. En este sentido, la Ilustración, la Aufklärung, es como un llamado, como una proclama al coraje, al valor de usar el propio entendimiento y no obedecer más a la autoridad por el hecho de ser tal. Pero es preciso recordar que no se trata de un llamado a la desobediencia en nombre de una autonomía primordial o esencial; la crítica en Kant es la pregunta formulada al saber sobre sus propios límites, a la razón sobre sus peligros, de ahí que la crítica en sentido kantiano diría:

... que nuestra libertad se juega menos en lo que emprendemos, con más o menos coraje, que en la idea que nos hacemos de nuestro conocimiento y de sus límites y que, en consecuencia, en lugar de dejar que otro diga "obedece", es en ese momento, cuando nos hayamos hecho del propio conocimiento una idea justa, cuando podremos descubrir el principio de la autonomía y cuando ya no tendremos que oír el obedece; o más bien, el obedece se fundará sobre la autonomía misma. (Foucault, 2007, p. 13)

La crítica implica una autonomía del sujeto, pero a su vez, la autonomía implica el reconocimiento de los límites del propio conocimiento, de la razón, de ahí que autonomía y obediencia no sean cuestiones antagónicas o excluyentes. La actitud crítica de Foucault se ubica, entonces, en la línea kantiana de la pregunta por la Ilustración; sin embargo, con una modificación de perspectiva. La crítica kantiana se orientaba hacia el conocimiento, se trataba de una "investigación sobre la legitimidad de los modos históricos del conocer"; en cambio, la actitud crítica de Foucault no se plantea el problema del conocimiento sino el problema del poder, es decir, se trata de señalar las "conexiones entre unos mecanismos de coerción y unos contenidos de conocimiento", de ahí, dice:

Lo que buscamos, entonces, no es saber lo que es verdadero o falso, fundado o no fundado, real o ilusorio, científico o ideológico, legítimo o abusivo. Buscamos saber cuáles son los lazos, las conexiones que pueden ser señaladas entre mecanismos de coerción y elementos de conocimiento, qué juegos de reenvío y de apoyo se desarrollan entre unos y otros, qué hace que tal elemento de conocimiento pueda tomar unos efectos de poder referidos, en un sistema tal, a un elemento verdadero o probable, incierto o falso, y lo que hace que tal procedimiento de coerción adquiera la forma y las justificaciones propias de un elemento racional calculado, técnicamente eficaz, etc. (Foucault, 1995, p. 26)

En otras palabras, la crítica tiene que ver con el análisis de las relaciones saber-poder, no del saber y del poder o de un saber y un poder como si fuesen "unos principios generales de realidad"; se trata más bien de dos términos que tienen una función metodológica, no son más que una rejilla de análisis. El saber hace referencia "a todos los efectos de conocimiento que son aceptables en un momento dado y en un dominio definido" (p. 26) y el poder a una "serie de mecanismos particulares, definibles y definidos, que parecen susceptibles de inducir comportamientos o discursos" (p. 27). La crítica no pretende, entonces, describir cómo el poder reprime o determina al saber, sino describir unos nexos saber-poder que hacen posible la "aceptabilidad de un sistema", como la enfermedad mental, la penalidad, la delincuencia, la sexualidad, la infancia o la educación pública.

Para efectos de procedimiento, Foucault identifica una dimensión arqueológica del análisis, centrada en las positividades discursivas, en los efectos de conocimientos aceptables en un momento dado, y una dimensión genealógica cuyo propósito es "restituir las condiciones de aparición de una singularidad a partir de múltiples elementos determinantes, de los que no aparece como el producto sino como el efecto" (p.31). De esta manera, la arqueología y la genealogía como herramientas de una actitud crítica no tienen como propósito desvelar los errores, la ilusión, las oscuras intenciones detrás del saber ni poner en evidencia el poder o las formas de dominación que se ocultan tras el saber: como actitud crítica vinculada a la tradición de la modernidad, de la Ilustración, la actitud de Foucault es más bien una decisión de salir de la minoría de edad, de hacerse una idea justa de nuestras verdades, de hacer uso de ciertas herramientas para determinar los límites de lo sabido, de 
lo conocido, de lo pensado, en últimas, una opción por no ser gobernado de cierta forma, en vista de ciertos propósitos y por medio de ciertos procedimientos que llegaron a convertirse en legítimos en el campo de las llamadas ciencias sociales y humanas.

Partiendo de estas herramientas, en investigaciones recientes ha sido posible llevar a cabo una arqueo-genealogía, no de la pedagogía sino de la racionalidad pedagógica moderna. Ello no implica la construcción de una pedagogía crítica, por ejemplo, que se opondría a una pedagogía tradicional o acrítica y que sería, por ello, una crítica más de la pedagogía. La crítica se centró en la descripción y análisis de las condiciones de posibilidad y funcionamiento de determinadas formas, medios y fines, en últimas, de ciertas prácticas que calificamos como pedagógicas y que dado su propósito de conducción de la conducta propia y de los otros podríamos también llamar prácticas de gobierno. No es que exista una racionalidad propia de la pedagogía, sino que en determinadas prácticas es posible describir una "racionalidad", es decir, unas reglas de producción y funcionamiento que están histórica y geográficamente delimitadas. Entonces, no se intentó, por ejemplo, una crítica a la didáctica comeniana por universalista o por desconocer la naturaleza infantil. Tampoco se criticó la educación liberal por su carácter ideológico, su falsa idea de libertad que escondía las intenciones de gobernar la infancia. Y a esas formas del pasado no se opuso una pedagogía crítica que procurara superar los efectos negativos para la constitución de subjetividades libres, autónomas o críticas. Lo que se intentó fue describir y analizar cómo eso que los científicos sociales llaman "modernidad" consiste en la creación y el desarrollo de técnicas de gobierno de todos y cada uno, técnicas que en su operación, en su actualización, se han transformado desde un modo que hemos llamado disciplinar hacia otro liberal y, más recientemente, otro neoliberal (Noguera, 2011a, 2011b, 2012, 2013; Noguera \& Marin, 2011).

No ha sido una descripción "neutra". Desde luego, la actitud crítica se orienta por la pregunta sobre el sentido de las formas en que nos gobiernan y en que nos gobernamos; por eso, las conclusiones a las que llegamos son un llamado a ejercer nuevas formas de conducción que no son ideales sino utópicas, o mejor, heterotópicas; es decir, implican la construcción de espacios paralelos o al margen de la tendencia neoliberal, conservadora $y$, aun, de las llamadas hasta ahora "críticas".

\section{Pedagogización, educacionalización}

Como con la gran mayoría de los asuntos que aquí se plantean, no somos los autores de esos conceptos de educacionalización o pedagogización. Para el caso nacional fue tal vez el profesor Mario Díaz quien, al final de su libro (tesis doctoral) sobre el campo intelectual de la educación en Colombia (Díaz, 1993), utilizó por primera vez esa expresión para referirse a las transformaciones contemporáneas del dispositivo pedagógico. Autores como Depaepe et al. (2008) señalan que fue el sociólogo alemán Janpeter Kob quien acuñó el término hacia finales de la década de 1950, y ya tres décadas después, una francesa, escribía un libro titulado La sociedad pedagógica (Beillerot, 1982), en el que analizaba algunas de las formas de pedagogización de la vida cotidiana de esos años que no han dejado de intensificarse y expandirse. Recientemente, Smeyers \& Depaepe (2008), profesores e investigadores belgas del campo de la educación, editaron un libro dedicado al tema de la "educacionalización", en el que resaltaban la importancia y pertinencia de esa categoría para el análisis de la expansión de la educación en el mundo occidental moderno.

Siguiendo algunos de esos autores podría definirse la pedagogización (o educacionalización) como una categoría para describir la orientación general de los procesos centrales y desarrollos de la historia de la educación, o como un concepto relacionado con la expansión cuantitativa y cualitativa de la intervención educacional y pedagógica en la sociedad moderna. $\mathrm{Si}$ bien para estos autores se trata de un asunto vinculado con la propia historia de la educación occidental en la Modernidad, podría decirse que durante el último medio siglo el fenómeno se ha vuelto más evidente, como lo demuestran las reflexiones de intelectuales de distintos campos de las ciencias sociales y humanas.

Por ejemplo, en 1993, Peter Drucker, filósofo y economista de origen austriaco lanzaba su libro $L a$ sociedad poscapitalista, en el cual anunciaba la llegada de un nuevo tipo de sociedad fundamentada en el conocimiento, es decir, se trataba de una sociedad en donde el recurso económico fundamental, el medio de producción fundamental, ya no eran ni la tierra ni el trabajo, sino el conocimiento, más particularmente, el conocimiento especializado. Desde luego, Drucker no estaba proponiendo una reivindicación del conocimiento en sentido humanista, el conocimiento de las humanidades, la cultura general que persiguió el bachillerato clásico europeo del siglo xIx y parte del xx. Se trata más bien de un know-how, de un saber 
hacer, de un conocimiento que se prueba a sí mismo en la acción, conocimiento que es información eficaz para orientar la acción, conocimiento enfocado hacia unos resultados (Drucker, 2004). En este sentido, la educación o la "persona educada", en palabras de Drucker, está en el centro de las preocupaciones de esa nueva sociedad y dada su importancia, la educación no puede ser más un monopolio de las instituciones escolares. La sociedad toda debe ser una sociedad educadora:

La educación en la sociedad postcapitalista tiene que saturar a toda la sociedad y a las organizaciones que dan empleo: las empresas, las oficinas del gobierno, las entidades sin ánimo de lucro, deben convertirse en instituciones de aprendizaje $y$ enseñanza, y las escuelas tienen que trabajar en asociación con los empleadores y las organizaciones que dan empleo. (Drucker, 2004, p. 270)

Unas décadas antes, E. Faure (1973), como presidente de una Comisión conformada por la Unesco para analizar las condiciones de la educación mundial en ese momento, acuñaba en su informe dos términos nuevos que posteriormente serían bastante conocidos en el medio educacional: ciudad educativa y educación permanente. Los investigadores anglosajones tradujeron el primero de ellos como learning society, sociedad del aprendizaje o sociedad aprendiente y el segundo término ha llevado a otros a pensar en la condición del ser humano contemporáneo como un lifelong learner, un aprendiz permanente o un cosmopolita inacabado (unfinished Cosmopolitan) según Popkewitz (2009).

Otros investigadores, como Simons y Masschelein (2008), utilizan la categoría "dispositivo de aprendizaje" para explicar la manera como funciona el liberalismo avanzado (neoliberalismo) en su condición de práctica de gobernamiento de la población, o dicho en otras palabras, para explicar cómo el liberalismo avanzado es una forma de gobernamiento que funciona a través del aprendizaje. Esto significa que como efecto de una compleja estrategia de saber-poder, un conjunto significativo de asuntos relacionados tradicionalmente con la acción de Estado son cada vez más considerados como problemas de aprendizaje. Así, el desempleo antes que un efecto estructural del sistema se ve como la falta de iniciativa (emprendimiento) o de competencias de los ciudadanos; los problemas de salud, considerados como efecto de los hábitos y prácticas de los individuos, requieren de aprendizajes o re-aprendizajes especializados sobre hábitos alimentarios, prácticas saludables y control de riesgos; la participación ciudadana, igualmente, es un asunto que involucra aprendizajes relacionados con el funcionamiento del Estado, con la veeduría, con la elaboración de presupuestos, con la rendición de cuentas, entre otros.

En fin, la pedagogización social contemporánea significa la centralidad de los procesos de aprendizaje en la vida cotidiana de las personas, la centralidad del saber, del conocimiento y de la información en las prácticas sociales, políticas y económicas; centralidad que ha generado una intensa y extensa proliferación de prácticas y discursos de carácter educacional manifiestos en un sinnúmero de "pedagogías". Esa proliferación de discursos y prácticas pedagógicas ha traído un doble efecto que podríamos calificar de paradójico y que se expresa en dos problemas centrales: de una parte, asistimos hoy a un debilitamiento del campo de saber pedagógico; de otra, la educación, tal como se entendió en la modernidad sólida (para hablar en términos de Bauman), se encuentra hoy en un impasse, en una sin salida o, por lo menos, en una encrucijada. A continuación vamos a ver en detalle estos dos asuntos.

\section{Debilitamiento de la pedagogía: exaltación de lo pedagógico}

Durante el siglo xIx y la primera mitad del siglo xx, la pedagogía adquirió sistematicidad y reconocimiento como disciplina. En el ámbito alemán, los trabajos de F. Herbart llevaron a considerar la Pädagogik como la ciencia de la educación, posteriormente se consolidó una tradición intelectual en la cual se desarrollaron distintas perspectivas (o paradigmas) como la pedagogía general o sistemática, la pedagogía de las ciencias del espíritu (Dilthey, Schleiermacher), la pedagogía crítico-reflexiva (Klafki), la ciencia de la educación crítica (Blankertz, Mollenhauer, etc.), la ciencia de la educación reflexiva (Lenzen), etc. ${ }^{1}$ En el ámbito francófono, se destacan las elaboraciones de los llamados filósofos pedagogos, como Marion y Compayré, la mirada desde la sociología de una ciencia de la educación de Durkheim, la psicopedagogía de Claparède, la pedagogía activa de Decroly y Ferrièrre, hasta las ciencias de la educación en el Instituto Jean Jacques Rousseau de Ginebra y, más tarde, en el ámbito francés, de Mialaret, Debesse y Château. En la tradición anglosajona, las propuestas de A. Bain de la educación como ciencia, los estudios curriculares como campo de investigación iniciado desde los trabajos de Bobbit y Charters, los trabajos de Dewey alrededor del concepto de educación

1 Sobre la Pedagogía alemana sugerimos consultar el trabajo del profesor Andrés Klaus Runge (2008). 
(educación y democracia, educación y experiencia, ciencia de la educación), hasta las elaboraciones de una teoría crítica de la educación en Carr y Kemmis.

En Colombia, al igual que en los demás países latinoamericanos, la consolidación de la pedagogía como disciplina no constituyó una tradición particular como en los casos europeo y anglosajón; sin embargo, es posible identificar con claridad, por lo menos hasta la década de 1990, diversos procesos e intentos de apropiación y elaboración más o menos sistemáticos sobre los problemas de la pedagogía y la educación. Desde las apropiaciones de Pestalozzi, la llamada pedagogía clásica de los hermanos Restrepo Mejía, la construcción de una pedagogía activa con el movimiento educacionista de las décadas de 1920 y 1930, la circulación (apropiación) y producción de conceptos y teorías en las escuelas normales, la Escuela Normal Superior y las facultades de educación, el Movimiento Pedagógico de 1980-90 (el Campo Intelectual de la Educación de Mario Díaz o el Campo conceptual de la pedagogía de Zuluaga y Echeverri) hasta las elaboraciones recientes sobre la(s) didáctica(s), la enseñanza de las ciencias y los saberes escolares, encontramos una amplia y diversa producción intelectual que hoy no encuentra los medios ni escenarios para una articulación y consolidación como campo de estudios.

Tanto las tradiciones europeas y anglosajonas, como la propia tradición nacional se han visto hoy relegadas ante la proliferación intensiva de discursos calificados de pedagógicos que aparecen en los más diversos espacios institucionales (empresas, escuelas, ONG, hospitales, cárceles, oficinas gubernamentales, movimientos sociales, partidos políticos, clubes deportivos, instituciones de salud o recreativas, etc.). Como una muestra de esta dispersión y proliferación, podríamos identificar, por lo menos, tres grandes tipologías en que se localizan las pedagogías contemporáneas:

1. Pedagogías para todo aquello que es necesario enseñar y aprender: pedagogía de la paz, de la felicidad, del ocio, de la esperanza, pedagogía ciudadana, de la autonomía, de la ecología, de la diferencia, de los medios, del cuerpo, de la sexualidad, del conocimiento, del trabajo, del deporte, de la salud, de la comunicación, de la imagen, de la economía y de todos los saberes y disciplinas.

2. Pedagogías según los sujetos, usuarios o destinatarios: pedagogías feministas, masculinas, de la infancia, de la tercera edad, de los oprimidos, de las negritudes o afro, de los trabajadores, de los adultos, etno-pedagogías, etc.
3. Pedagogías según la cualidad o filiación: pedagogías participativas, nuevas, tradicionales, innovadoras, culturales, interculturales, contemporáneas, posmodernas, no-directivas, críticas, participativas, correctivas, hospitalarias, constructivistas, liberales, poscoloniales, curativas, preventivas, ascéticas, hedonistas, etc.

Como efecto de esa proliferación, los tradicionales sujetos vinculados con ese campo de saber (o con esa disciplina, para el caso de las tradiciones europeas y anglosajonas), se han visto desplazados por nuevos "pedagogos" o "formadores" o "educadores" informales que toman diversas formas: entrenadores (de equipos deportivos o personales, personal trainers, coaches), pastores, publicistas, guías espirituales, consejeros, terapistas, periodistas, etc. Una multiplicidad de profesionales asume actividades catalogadas como pedagógicas o educativas o formativas o de enseñanza, mientras los tradicionales encargados de tales actividades pierden cada vez más legitimidad, reconocimiento social y profesionalismo.

\section{El impasse de la educación (y de la pedagogía)}

El debilitamiento de la pedagogía (de las tradiciones pedagógicas modernas) y la exaltación de lo pedagógico está íntimamente relacionado con lo que podríamos denominar el impasse de la educación. Para comprender mejor lo que quiere decir esa expresión, es necesario recordar que el concepto de educación es un concepto moderno formado durante el siglo XVII, pero desarrollado a partir de las elaboraciones de Rousseau y Kant, retomadas a su vez a comienzos del siglo xx por los llamados pedagogos activos (Noguera, 2012). Tal concepto se fundamentaba en el reconocimiento de la precariedad de nuestra condición inicial al nacer, en nuestra necesidad más o menos prolongada de otros para nuestra supervivencia, en nuestra capacidad de perfeccionamiento, de ahí que Kant señalara que "El hombre es la única criatura que ha de ser educada" (2003, p. 29).

Para Kant, la educación implicaba la posibilidad de transformación de la animalidad en humanidad, lo que significaba que era ella la principal herramienta para la conformación del "hombre" que nacía inacabado y frágil. La condición de la educación era, entonces, la existencia de un adulto y un infante o joven (igualmente lo planteó Durkheim hacia finales del siglo XIX); se trataba de una actividad jerarquizada en donde uno dirige al otro con el propósito final de que ese otro consiga, como parte de esa dirección, su propia autonomía, es decir, alcance su propio 
autogobierno: gobernar, dirigir, conducir para que el otro aprenda a conducirse. Décadas antes de Kant, aunque en una perspectiva diferente, Rousseau señalaba la misma idea de necesidad de perfección del ser humano a través de la educación. Si bien Emilio no debía asistir a la escuela para conseguir una adecuada educación, la guía y el acompañamiento de su gouverneur (como lo llamaba Rousseau) y un medio natural (el campo) adecuado por este para el crecimiento, desarrollo y perfeccionamiento, eran las condiciones para su educación. Es ese gobernador o conductor un adulto que responsable, dedicada y amorosamente destina varios años de su vida a la tarea de educar al "recién llegado"; y si bien esa tarea la realiza fuera de la ciudad (de la sociedad de su época), el propósito es devolverlo a ella plenamente formado para que sea ejemplo del nuevo ciudadano.

Tanto Emilio como el discípulo de Kant estaban destinados a ser seres libres y autónomos y ello sólo era posible a través de la obediencia: a las leyes naturales, en el caso del primero; al adulto que representaba la razón, en el otro. Y si bien la educación de Emilio partía de un principio de libertad (libertad de ejercitarse, libertad de actuar por su propio interés), tal era una libertad "bien regulada" en la medida en que el ayo (gouverneur y no preceptor) tenía una meta para Emilio y adaptaba el medio para que fuese de aquel de quien recibiese las lecciones. En los inicios del siglo Xx, el pedagogo francés G. Compayré proponía en su Curso de pedagogía (1920) esta fórmula:

La educación es el conjunto de los actos reflexivos por medio de los cuales se ayuda a la naturaleza en el desarrollo de las facultades físicas, intelectuales y morales del hombre, para buscar su perfección, su felicidad y la realización de su destino social. (p.18).

Esos actos reflexivos por medio de los cuales se ayudaba a la naturaleza al desarrollo de sus facultades sólo podían ser asunto de adultos.

De esta forma queda claro que la idea moderna de educación implicaba la existencia de un adulto que representaba la mayoría de edad, el uso adecuado de la razón (Kant) o el conductor o guía (Rousseau) que dirigía (y no simplemente acompañaba) el crecimiento y desenvolvimiento de las fuerzas naturales de Emilio que era un infante. Adulto e infante: esa era la condición para la educación. Hoy, como lo muestra Postman (1999) y lo sugiere Narodowski (1999), la infancia se acabó y con ella, la propia educación entra en crisis. En su escrito sobre la "crisis de la educación" Arendt (1996) ya anunciaba este fenómeno en el seno de la sociedad norteamericana de mediados de siglo xx como un efecto de "ese complejo de teorías educativas modernas que nacieron en Europa central y consisten en una notable mezcolanza de sensatez e insensatez que pretendía lograr, bajo el estandarte de una educación progresista, una revolución radical en todo el sistema educativo" (p. 190). Al auge de esas teorías (fundamentadas en el romanticismo rousseauniano) se sumaron la crisis de la idea de autoridad y el auge del concepto de igualdad que llevó a igualar o borrar, en la medida de lo posible, "las diferencias entre jóvenes y viejos, entre personas con talento y sin talento, entre niños y adultos y, particularmente, entre alumnos y profesores" (p. 192).

El entusiasmo contemporáneo en las libertades, la mirada ingenua sobre el interés del sujeto, sobre su autonomía y sus derechos (casi naturales) forman parte del bloqueo de la educación, pero a la vez, como lo mostró Noguera (2013), constituyen los fundamentos de la tentativa actual de una forma de gobernamiento que podríamos calificar, siguiendo a Foucault (2007), de neoliberal. Esta manera de conducir los individuos requiere para su funcionamiento de la promoción de determinados procesos de subjetivación según los cuales el individuo es poseedor de una capacidad de agencia, de una libertad de acción y elección, de unos determinados intereses y capacidades que debe desarrollar; en últimas, de un "yo" o personalidad particular que a la vez que lo hace singular y único, es el fundamento para su perfeccionamiento y la consecución de su felicidad.

Pero a diferencia de la manera liberal de gobernar (tipo educación en Rousseau e incluso en los llamados pedagogos activos) el agente o la capacidad de agencia del individuo contemporáneo parece haberse desprendido de su relación con el medio y en ese sentido, el concepto de interés como inter-esse se ha convertido en una propiedad del individuo, o mejor, del yo agente, algo que forma parte de su esencia e identidad. El papel que en la teoría darwiniana tenía el medio o el ambiente como agente del cambio evolutivo, ahora, en el concepto de aprendizaje permanente y a lo largo y ancho de la vida, se le atribuye al mercado que, independientemente de las condiciones sociales, políticas, económicas, culturales, actúa como el acicate que mueve al individuo a la acción. El aprendiz permanente es el homo economicus, el empresario de sí mismo que gerencia su propio capital para responder a las demandas del medio (mercado). Para la gramática del aprendizaje permanente, el medio es el mercado, y este funciona como una segunda naturaleza en la que los individuos interactúan (o compiten) para ser exitosos y felices.

El problema, en términos de gobernamiento o en términos antropotécnicos (Sloterdijk, 2012), es la asociación que implica el aprendizaje con el inte- 
rés, con la libertad y con los derechos. Aquí vale la pena resaltar el hecho de que el gobierno neoliberal requiere de un mecanismo del dispositivo de soberanía: los derechos individuales, sin ellos no podría funcionar, pues el empresario de sí mismo es un sujeto de derechos, un sujeto que tiene el derecho de elegir (consumir), de desarrollar libremente su personalidad, de preservar su intimidad. Así, el interés pasó de ser ese inter-esse, es decir, de estar entre el individuo y el medio a ser un asunto de propiedad e identidad del "yo": como "yoes", tenemos intereses, estamos constituidos por intereses los cuales tenemos derecho de satisfacer. Por otro lado, la libertad pasó de ser "bien regulada" a desregulada casi completamente, pasó de ser una meta, un objetivo, a ser la condición, el principio para la "educación", o mejor, para el aprendizaje del individuo. Y aquí es donde la educación en su sentido clásico y liberal, se hace imposible hoy, aquí es donde la crisis de la educación encuentra su punto crucial.

El diagnóstico anterior puede ser corroborado con los análisis realizados por Sloterdijk (2102) a propósito del análisis sobre las antropotécnicas ${ }^{2}$ contemporáneas. En la perspectiva trazada por este filósofo alemán, lo humano no sólo estaría dado por una configuración biológica - condición fundamental, pero a la vez insuficiente - sino, además, por el efecto de una antiquísima tradición de ejercicios físicos y mentales que, como especie, hemos puesto en práctica en diferentes sociedades a través del tiempo. Tal conjunto de técnicas o ejercicios atiende a un principio de elevación de la vida propia respecto a modos de vida anterior y a la de otros seres humanos: se trata del imperativo metanoético que significa un impulso hacia el mejoramiento a través de la ejercitación. Según el filósofo alemán, durante muchos siglos ese imperativo fue potestad de grupos específicos, como grupos ascéticos y comunidades religiosas, que establecieron formas de vida en torno de esos ejercicios, constituyendo reglas para orientar -y cuando era necesario, reorientar - el modo de practicar la vida de sus integrantes.

Con la modernidad, o mejor, con la emergencia de la escolarización, hubo dos cambios trascendentales en el modo de operar de ese imperativo: en primer lugar, un énfasis en la necesidad de un cambio de vida desde el inicio mismo de esta (es decir, desde la infancia) y ya no sólo durante la edad adulta; en segundo lugar, el imperativo pasó de ser asunto de

2 "Entiendo, con esta última expresión, los procedimientos de ejercitación, físicos y mentales, con los que los hombres de las culturas más dispares han intentado optimizar su estado inmunológico frente a los vagos riesgos de la vida y las agudas certezas de la muerte" (Sloterdijk, 2012, p. 24). unos que voluntariamente lo asumían, a convertirse en una oferta extendida masivamente. La instrucción generalizada - que se consolidará sólo hasta finales del siglo XIX con la constitución de sistemas de instrucción pública nacionales - constituye un acontecimiento central para la modernidad, transformando las sociedades en asociaciones de ejercitación, de fitness, a través de una "alianza disonante" entre escuela y Estado con dos facetas claramente definidas: 1) la institución escolar se convirtió en un mecanismo estatal para el gobierno de poblaciones e individuos; 2) la pedagogía escolar produjo - al margen de la pedagogía estatalizada - un conjunto de excedentes y efectos inesperados que han irrumpido en la cultura moderna, y que fueron posibles en tanto la escuela pudo ser un escenario de retirada del mundo. No en vano se advierte que "quien apueste por los pedagogos para obtener ciudadanos tendrá que contar con efectos inesperados" (Sloterdijk, 2012, p. 433).

Para Sloterdijk (2012, p. 549), en la actualidad la posición de la escuela en esa alianza se habría desdibujado, al convertirse en una especie de sistema autorreferencial vacío, cuyo problema

[...] consiste palmariamente en que no sólo ya no es capaz de cumplir con el encargo que recibe del Estado de formar ciudadanos, al devenir demasiado poco clara la definición del fin ante las exigencias del mundo profesional actual, sino que tal problema se articula con una claridad aún mayor en el abandono de su excedente humanístico y artístico, para dedicarse a una actividad más o menos desespiritualizada de rutinas didácticas con un fundamento pseudocientífico.

En palabras de Simons y Masschelein (2014) la escuela y su potencial habrían tratado de ser domesticados, es decir, puestos al servicio de fines que no necesariamente les correspondían, en especial desde mediados del siglo xx. Una parte de esa domesticación proviene del exterior de la escuela, como cuando se le encarga la solución de los problemas sociales existentes (politización), cuando asume funciones de cuidado individual que tradicionalmente le corresponden a la familia (familiarización) o cuando se asumen supuestos sobre las diferencias naturales en cuanto a inteligencia y capacidad de los estudiantes, que operan como una suerte de mecanismo de selección natural (naturalización). De otra parte, hay elementos de esa domesticación cuya proveniencia es interior, como la concentración de las prácticas escolares en la eficiencia y la eficacia (tecnologización), la sustitución de la enseñanza por una terapéutica que se ocupa del bienestar y la motivación para aprender (psicologización), y la infantilización continua producida por métodos que 
contrarrestan el aburrimiento - como los programas de entretenimiento - pero no fomentan el estudio y el esfuerzo (popularización).

\section{Retomar la crítica, actualizar la crítica}

Volviendo al inicio de este texto, habría que decir que después de las teorías críticas derivadas de las perspectivas marxistas, las elaboraciones de Foucault pueden constituirse en nuevas herramientas para potenciar la crítica de los asuntos educacionales y pedagógicos contemporáneos, particularmente aquellos relacionados con lo que se ha señalado como el impasse de la educación que no es otra cosa que una crisis del gobierno de todos y cada uno. Podría decirse que las herramientas del filósofo francés posibilitan hoy una crítica de la crítica, y esto es central en un momento en el cual la crítica al papel del Estado y a los dispositivos disciplinarios, por ejemplo, así como la exaltación de la libertad o de las libertades individuales son valorizadas tanto por el pensamiento de las izquierdas como por los neoliberales. Pensar de otro modo hoy significa hacer una crítica a la individualización, al individuo (iy no solo al individualismo!); significa hacer una crítica a la idea de libertad, es decir, estudiar su constitución histórica, sus condiciones de posibilidad y su significado en términos gubernamentales. He ahíla potencia del pensamiento de Foucault (y también de Sloterdijk), en particular, la importancia de nociones como las de gobernamiento y antropotécnicas, herramientas analíticas para una "ontología del presente".

Para terminar quisiéramos destacar un par de asuntos que pueden resultar potentes en esta línea: 1) Frente al cambio en el lenguaje educativo, si bien puede parecer un asunto superfluo para algunos, en realidad es un tema fundamental. De una parte, en esa proliferación y dispersión de las "pedagogías contemporáneas" suelen emplearse de manera no diferenciada los conceptos propios de la pedagogía, sin reconocer la tradición de pensamiento que en estos se inscribe; así, conceptos como educación, formación, pedagogía, aprendizaje, entre otros, son tratado como sinónimos. De otra parte - y como contracara de lo anterior - la centralidad del concepto de aprendizaje para expresar y definir asuntos educativos y pedagógicos, instrumentaliza cuestiones educativas y formativas a la vez que diluye otras centrales para pensar el problema de lo humano como asunto de construcción o creación. Aunque no todas las perspectivas sobre el aprendizaje conducen a esa reducción (algunas de ellas han permitido comprender aspectos importantes en relación con el sujeto que es educado), cuando el objetivo que se traza es la satisfacción de necesidades de aprendizaje —incluso si se expresan como derechos- la educación se torna en una especie de transacción económica, donde el maestro es un proveedor de servicios y el aprendiente (antes estudiante) es un usuario (Biesta, 2013). En ese sentido, es preciso volver a esa idea de educación como una forma de humanización - lo que reconectaría con esa larga tradición del imperativo metanoetico y la ejercitación-, pero entendida como parte de una construcción histórica, no como a priori universal. Algo semejante puede plantearse en torno a la idea de razón, ya no tanto como característica inherente a la naturaleza humana, sino como efecto de una contingencia histórica que hizo posible y aún permite abrir la pregunta por lo humano.

En segundo lugar, es importante considerar el tipo de ejercicios que se proponen hoy en los escenarios educativos, particularmente en la tan criticada escuela. Debe quedar claro que no se trataría de facilitar las cosas para hacer sentir bien a los estudiantes, sino de generar esfuerzo, tensión, interés por estudiar (lo cual tampoco significa que ir a la escuela sea garantía de tedio). Sobre este punto, muchas de las perspectivas que se autodenominan críticas hoy claman por experiencias y contenidos educativos sensibles a los contextos sociales y las necesidades individuales, más semejantes a la cotidianidad, a la vida social. En esta línea, una demanda que se le hace a las instituciones escolares es la generación de currículos flexibles, que de entrada sean inter o multidisciplinares, que reconozcan la multiplicidad de los saberes y promuevan su intercambio. Frente a esto, es pertinente recordar que el "currículo escolar" constituye, en mayor o menor medida, aquello común que se produce o se pone a circular en la escuela. En esta perspectiva, concordamos con el planteamiento de M. Young (2011), quien, frente "a la retórica hueca de mucho de lo que pasa por pedagogía crítica" (p. 609), destaca el lugar de las disciplinas escolares en el currículo, cuya finalidad es la promoción del desarrollo intelectual de los estudiantes, no su motivación o la solución de problemas sociales. Esto también es importante para los profesores (en formación y en ejercicio), pues el conocimiento de su disciplina de enseñanza sirve de fundamento para el ejercicio de la autoridad, y también para los estudiantes, pues las disciplinas se convierten en generadoras de identidad

El mundo cotidiano no es como la escuela. No se divide en materias o disciplinas. Ese papel generador de identidad de las disciplinas es particularmente importante para alumnos de lugares desfavorecidos y para sus profesores. Muchos de esos alumnos llegaron a la escuela con poca experiencia 
de tratar el mundo como más que un conjunto de experiencias, en otras palabras, conceptualmente. Las disciplinas, con sus fronteras para separar aspectos del mundo que fueron probados a lo largo del tiempo, no sólo ofrecen la base para analizar y hacer preguntas sobre el mundo, también ofrecen a los estudiantes una base social para un nuevo conjunto de identidades como aprendices. Con las nuevas identidades referentes a las disciplinas, que los estudiantes adquieren por el currículo, acrecentadas a aquellas con que vinieron a la escuela, ellos tienen más probabilidades de ser capaces de resistir al sentido de alienación de sus vidas cotidianas fuera de la escuela, o al menos lidiar con él, la escuela puede promover tal capacidad (Young, 2011, p, 617) ${ }^{3}$.

\section{Referencias bibliográficas}

Arendt, H. (1996) La crisis en la educación. En: Entre el pasado y el futuro. Ocho ejercicios sobre la reflexión política. (pp. 185-208). Barcelona: Península.

Beillerot, J. (1982). La société pédagogique. Action pédagogique et controle social. París: Presses Universitaires de France.

Biesta, G. (2013) Para além da aprendizagem: Educação democrática para um futuro humano. Belo Horizonte: Autentica.

Compayré. G. (1920). Curso de pedagogía. México: Librería de la V. de Ch. Bouret.

Depaepe, M.; Herman, F.; Surmont, M.; Gorp, A.; Simon, F. (2008). About pedagogization: From the perspective of the History of Education. En Educational research: The educationalization of social problems (pp. 13-30). Springer, 2008.

Díaz, M. (1993). El campo intelectual de la educación en Colombia. Cali: Universidad del Valle.

Drucker, P. (2004). La sociedad poscapitalista. Bogotá: Norma.

Durkheim, E. (2003). Educación y sociología. Barcelona: Península.

Faure, E. (1973). Aprender a ser. La educación del futuro. Madrid: Alianza-Unesco.

Foucault, M. (1995) ¿Qué es la crítica? [Crítica y Aufklärung] Daimon. Revista de filosofía, No. 11. Murcia: Universidad de Murcia. 5-25. [Traducción de Javier de la Higuera].

3 La traducción al español es una traducción libre bajo nuestra responsabilidad. Aunque la fuente citada está en portugués, también se revisó el original en inglés.
Foucault, M. (2007). El nacimiento de la biopolítica. Buenos Aires: FCE.

Kant, I. (2003) Pedagogía (4⿳ạ Edición). Madrid: Ediciones Akal.

Narodowski, M. (1999) Después de clase. Desencantos y desafíos de la escuela actual. Buenos Aires: Ediciones Novedades Educativas.

Noguera, C. (2011a). Pedagogia e governamentalidade ou Da Modernidade como uma sociedade educativa. Belo Horizonte: Autêntica.

Noguera, C. (2011b). A governamentalidade nos cursos do professor Foucault. En G. Castelo-Branco, A. Veiga-Neto. Foucault, filosofia \& politica (pp. 71-80). Belo Horizonte: Autêntica.

Noguera, C. (2012). El gobierno pedagógico. Del arte de educar a las tradiciones pedagógicas. Bogotá: Siglo del Hombre.

Noguera, C. (2013). Crisis de la educación como crisis de gobierno. Sobre la ejercitación del animal humano en tiempos neoliberales. En Revista Colombiana de Educación, 65, 43-60.

Noguera, C. \& Marín, D. (2011). Educar es gobernar. En A. Cortés y D. Marín (comps.) Gubernamentalidad y educación. Discusiones contemporáneas (pp. 127-138). Bogotá: IDEP.

Popkewitz, T. (2009). El cosmopolitismo y la era de la reforma escolar. Madrid: Morata.

Postman, N. (1999) O desaparecimento da infância. Rio de Janeiro: Graphia.

Runge, A. (2008). Ensayos sobre pedagogía alemana. Bogotá: Universidad Pedagógica Nacional.

Silva, T. T. (1993) Deconstruindo o construtivismo pedagógico. Em: Educação e Realidade 2(18), 3-10.

Simons, M. \& Masschelein, J. (2008). It makes us believe that is about our freedom: notes on irony of the learning apparatus. En Educational research: the educationalization of social problems (pp. 191-204). Springer.

Sloterdijk, P. (2012). Has de cambiar tu vida. Valencia: Pre-Textos.

Smeyers, P. \& Depaepe, M. (2008). Educational research: The educationalization of social problems. Springer.

Young, M. (2011) O futuro da educação em uma sociedade do conhecimento: o argumento radical em defesa de um currículo centrado em disciplinas. Revista Brasileira de Educação, 16(48), Set.-Dez. 2011.609-625. [Traducción al português de Laura Beatriz Áreas Coimbra].

Veiga-Neto, A. (Comp.) (1997) Crítica pos-estructuralista y educación. Barcelona: Laertes. 